\title{
Infrastructure planning for Carbon Capture and Storage
}

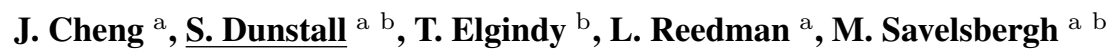 \\ ${ }^{\text {a } C S I R O ~ E n e r g y ~ T r a n s f o r m e d ~ F l a g s h i p ~}$ \\ ${ }^{\mathrm{b}}$ CSIRO Mathematics Informatics and Statistics \\ Email: Simon.Dunstall@csiro.au
}

\begin{abstract}
Carbon capture and storage (CCS) is seen as one of the key technologies for cutting $\mathrm{CO}_{2}$ emissions from coal power plants. It is proposed that carbon capture and storage could reduce $\mathrm{CO}_{2}$ emissions to the atmosphere from a modern power plant by approximately $80-90 \%$. Building and operating infrastructure for capturing, transporting, and storing $\mathrm{CO}_{2}$, however, is forecast to be expensive.

To gain a better understanding of and to develop clearer insight into the cost of introducing CCS in Australia, the CSIRO Infrastructure Futures Analysis Platform has been extended with capabilities to handle pipeline infrastructures and with an optimisation module specifically designed for CCS infrastructure planning. The Infrastructure Futures and Analysis Platform is a software system for addressing questions regarding the optimal selection, configuration and deployment of infrastructure.
\end{abstract}

This paper has two objectives: (1) To introduce an optimisation model for the detailed design and operation of a carbon capture and storage network and present the results of a computation study involving eastern Australia. (2) To introduce the Infrastructure Futures and Analysis Platform and demonstrate its extendability and versatility by discussing what was needed to customise it for use in the carbon capture and storage context.

The core of the carbon capture and storage optimisation module is a multi-period network design model. Given yearly, system-wide carbon capture targets, the model decides when and where to build carbon capture facilities, open basins for geo-sequestration, and install pipelines connecting capture sites with carbon sinks so as to minimise the total cost over the planning horizon.

The preliminary results of the computational study are intended primarily to validate the optimisationbased approach and to provide the basis for a more extensive study investigating different scenarios involving different and larger regions, different system-wide carbon capture targets, and different economic costing models.

Keywords: Infrastructure planning, carbon capture and storage, integer programming 


\section{INTRODUCTION}

Carbon capture and storage (CCS), alternatively referred to as carbon capture and sequestration, is seen as one of the key technologies for cutting $\mathrm{CO}_{2}$ emissions from coal power plants, thus mitigating the contribution of fossil fuel emissions to global warming (International Energy Agency (2010)). CCS applied to a modern conventional power plant has the potential to reduce $\mathrm{CO}_{2}$ emissions to the atmosphere by approximately 80-90\% compared to a plant without CCS (Metz et al. (2005)). Currently, building and operating infrastructure for capturing, transporting and storing $\mathrm{CO}_{2}$ is a largely untested proposition and is expected to be expensive. However, it is forecast that coal with CCS electricity generation could be economically competitive in the 2025-2030 timeframe in Australia (Coal Utilization Research Council (2005)).

To gain a better understanding of the potential costs of introducing CCS in Australia, we have augmented the Infrastructure Futures Analysis Platform (IFAP) with capabilities to handle pipeline infrastructures and incorporated an optimization module specifically designed for CCS infrastructure planning. IFAP is a software system originally created to address transport infrastructure network design in order to fulfil regional freight transport demand.

At the heart of the CCS optimisation module is a multi-period network design model. Given yearly, system-wide carbon capture targets, the model decides when and where to build carbon capture facilities, open basins for geo-sequestration, and install pipelines to connect capture sites with carbon sinks so as to minimise the total cost over the planning horizon. We have employed a deterministic optimisation model to assess the cost of building and operating a CSS network. Deterministic optimisation models have obvious limitations, and in future work it will be beneficial to consider stochastic models. This would allow, for example, capturing the uncertainty in the storage capacity of a basin.

Our work involved extending the IFAP framework with entities specific to the CCS infrastructure, and forming an XML-based interface to an optimisation model (i.e., exporting input data from the IFAP database and importing the results into this database). A significant and time-consuming task was gathering and synthesizing the data for the computational study from various sources. The computational study considers only coal-fired power stations and geological sequestration along the coast of eastern Australia. The preliminary results of the computational study presented in this paper are intended primarily to validate the optimisation-based approach. Future work will investigate larger regions, different economic costing models and more complex system-wide carbon capture targets.

A variety of economic models have been developed to understand and predict changes in the way countries and regions produce and use energy, and to investigate issues such as the uptake of new technologies in electricity generation and the energy generation technology mix required to meet emissions reduction targets. These economic models use estimates of the cost of technologies: for example, estimates of the cost of carbon capture and storage on a per-MW basis. The quality of these estimates has an impact on the results produced by the models. With our CCS optimisation model we aim to provide more accurate cost estimates for use in such models. The potential for improved accuracy comes from directly considering the CCS network and its construction over time.

The remainder of this paper is organised as follows. In Section 2, we discuss the carbon capture and storage optimisation model. In Section 3, we present an overview of the IFAP system and discuss how the carbon capture and storage problem can be represented in the IFAP modeling framework. In Section 4, we present the results of our computational study for eastern Australia. Finally, in Section 5, we offer some concluding remarks.

\section{CARBON CAPTURE AND STORAGE OPTIMIZATION MODEL}

An integer programming model minimises the cost of building and operating a CCS network so as to meet yearly carbon capture targets. Carbon dioxide is captured and compressed at power plants, transported through pipelines to storage sites and sequestered in carbon sinks. The annual system-wide capture targets are given as input parameters for each year in the planning horizon. To ensure that the optimisation always provides valuable information, we allow for solutions that do not reach the capture target every year, but penalize such solutions heavily so that this only occurs when it is infeasible to reach the target in the years 
where shortfall occurs. The model assumes that for each operational capture facility, a maximum amount of carbon dioxide can be captured each year and a minimum amount must be captured each year. Building carbon capture facilities at a power plant has a one-time construction cost and a yearly operating cost which is calculated per unit of carbon captured at the site each year following construction. The model assumes that a carbon sink has a maximum storage capacity and a maximum yearly injection capacity. Building a carbon storage facility has a one-time building cost and a yearly operating cost calculated per tonne of carbon injected. It is possible to build pipelines of different diameters. The diameter of a pipeline determines its transport capacity. Building a pipeline has a one-time building cost and a fixed yearly maintenance cost incurred every year after the pipeline has been built. The integer programming formulation is given below:

\section{Parameters:}

$T$ : Set of time periods

Cap: Capture Sites

Stor: Storage Sites

$D$ : Pipeline types (i.e., diameters)

$E$ : Pairs of sites between which pipelines can be build to transport compressed carbon dioxide

$T_{t}$ : Target amount of $\mathrm{CO}_{2}$ in time period $t$

$P_{t}$ : Per-unit penalty for not meeting capture target at time $t$

$C_{e}^{d}$ : Cost of building a pipeline of type $d$ at edge $e$

$c_{e}$ : Cost of operating a pipeline at $e$ (for one time period)

$U^{d}$ : Maximum transport capacity of a pipeline of type $d$

$B_{c}$ : Cost of building capture site $c$

$B_{s}$ : Cost of building storage site $s$

$o_{c}$ : Cost of operating capture site $c$ (for one time period)

$i_{s}$ : Cost of injecting a tonne of carbon at storage site $s$

$U_{s}$ : Storage space at storage site $s$

$u_{s}$ : Injection capacity at storage site $s$ per year

$u_{c}$ : Capture capacity at capture site $c$ per year

\section{Decision variables:}

$x_{e}^{t}$ : flow through edge $e$ at time $t$

$z_{e}^{d t}$ : whether edge $e$ with diameter $d$ exists at time $t$

$s_{t}$ : shortfall at time $t$

$v_{c}^{t}$ : carbon captured from capture site $c$ at time $t$

$w_{s}^{t}$ : carbon injected into storage site $s$ at time $t$

$y_{c}^{t}:$ whether capture site $c$ exists at time $t$

$y_{s}^{t}:$ whether storage site $s$ exists at time $t$

\section{Minimize:}

$$
\begin{gathered}
\underbrace{\sum_{\text {pipe maintainance costs }} P_{t} s_{t}}_{\text {shortfall penalty }}+\underbrace{\sum_{e \in E, t \in T, d \in D} c_{e} z_{e}^{d t}}_{\text {pipe building costs }}+\underbrace{\sum_{e \in E, t \in T, d \in D} C_{e}^{d}\left(z_{e}^{d t}-z_{e}^{d(t-1)}\right)}_{\text {capture facility building costs }}+\underbrace{\sum_{\text {storage facility injection costs }} B_{c}\left(y_{c}^{t}-y_{c}^{(t-1)}\right)}_{\text {capture facility operating costs }}+ \\
\underbrace{\sum_{s \in \text { Stor }, t \in T} B_{s}\left(y_{s}^{t}-y_{s}^{(t-1)}\right)}_{\text {storage facility building costs }}+\underbrace{\sum_{c \in \text { Cap }, t \in T} o_{c} v_{c}^{t}}_{\text {capter }}+\underbrace{\sum_{s} w_{s}^{t}}_{t \in T, s \in \text { Stor }}
\end{gathered}
$$

\section{Subject to:}

$$
\begin{array}{r}
\sum_{c \in C} v_{c}^{t}=T_{t}-s_{t} \forall t \in T \\
v_{c}^{t} \leq u_{c} y_{c}^{t} \forall t \in T, \forall c \in C a p \\
v_{c}^{t} \geq l_{c} y_{c}^{t} \forall t \in T, \forall c \in C a p \\
w_{s}^{t} \leq u_{s} y_{s}^{t} \forall t \in T, \forall s \in \text { Stor }
\end{array}
$$




$$
\begin{array}{r}
\sum_{t \in T} w_{s}^{t} \leq U_{s} \forall s \in \text { Stor } \\
\sum_{e=(k, c) \in E} x_{e}^{t}+v_{c}^{t}=\sum_{e=(c, k) \in E} x_{e}^{t} \forall t \in T, \forall c \in \text { Cap } \\
\sum_{e=(k, s) \in E} x_{e}^{t}=w_{s}^{t}+\sum_{e=(s, k) \in E} x_{e}^{t} \forall t \in T, \forall s \in \text { Stor } \\
x_{e}^{t} \leq \sum_{d \in D} U^{d} z_{e}^{d} \forall t \in T, \forall e \in E \\
z_{e}^{d t} \geq z_{e}^{d(t-1)} \forall t \in T, \forall d \in D, \forall e \in E \text { with } z_{e}^{d 0}=0 \\
y_{c}^{t} \geq y_{c}^{(t-1)} \forall t \in T, \forall c \in \text { Cap } \text { with } y_{c}^{0}=0 \\
y_{s}^{t} \geq y_{s}^{(t-1)} \forall t \in T, \forall s \in \text { Stor } \text { with } y_{s}^{0}=0
\end{array}
$$

Constraint (1) recognizes any shortfall from the target capture amount in a period. Constraints (2) and (3) ensure that the amount captured at a site is between the lower and upper limits of a constructed capture facility. Constraint (4) ensures that the amount injected into a constructed capture site is less than its yearly injection capacity. Constraint (5) ensures that the total amount stored at a site during the planning horizon does not exceed the available storage capacity. Constraints (6) and (7) ensure flow balance at capture and storage sites, respectively. Constraint (8) ensures that compressed carbon flows between two locations only if a pipeline has been built and that the amount that flows can be accommodated by the pipe's diameter. Constraints (9), (10), and (11) establish that once infrastructure has been built it cannot be decommissioned during the planning horizon.

In this formulation the cost of constructing a section of pipeline between two points is directly specified in the input data (i.e., $C_{e}^{d}$, where $e$ refers to an edge in the graph of potential pipeline sections, and $d$ is the chosen pipe diameter). There is no relationship assumed between the cost of a pipeline on one edge and the cost on any other edge. This generally makes the model hard to solve because capacity is discretized and determined by way of the integer variables $z_{e}^{d t}$ (rather than, for example, by capacity variables with continuous real-valued domains which are multiplied by constant coefficients in cost equations). This, however, is a key feature of the model and is a necessity in realistic infrastructure planning problems. Furthermore, the actual route taken between the endpoints of a pipe section is relevant to costing but irrelevant to the optimisation problem as formulated: thus the route can be represented in arbitrary detail in the GIS user interface but it does not feature in the optimisation model.

The true cost of a pipeline (or any other transport corridor) between two points is a complex function of potentially many factors. Most of these factors would vary spatially and be very difficult to represent in linear mathematical models. Some factors could be hard to quantify at all. Consider the datasets that might be available to a future (hypothetical) CCS network planning team. The potential-pipelines data held by this team will tend to be made up of some relatively vaguely-defined pipeline sections, some sections associated with detailed engineering analyses and public consultation insights, and other sections defined with a rigour that lies between these extremes. A useful infrastructure modelling system has to support this kind of heterogeneity. In terms of infrastructure construction costs, our formulation supports this by using discrete capacity options and "externally" computed costs. The user interface to this model, the IFAP System, provides means for specifying the costs: either by way of entering pre-computed costs per pipeline section, or by applying "templates" for different types of pipeline. This is an approach that is in common with IFAP's original freight transport network design application.

\section{INFRASTRUCTURE FUTURES ANALYSIS PLATFORM}

The purpose of the IFAP system is to address questions regarding the design of network infrastructure to support time-varying multi-commodity demand in a region. IFAP was originally formulated for freight network planning. IFAP consists of a database, optimisation solvers and a user interface embedded in a Geographic Information System. IFAP solvers seek to minimise the cost of infrastructure provision subject to fulfilling multi-commodity transport demand.

A real-world transport system is modelled in IFAP using five basic entities: facilities, links, commodities, 
vehicles, and processes:

- Commodities: Commodities represent the materials and/or goods that are produced, transported, and consumed in the system. Each commodity is identified by a name (e.g., copper concentrate, $\mathrm{CO}_{2}$, passengers), a density and a set of requirements and restrictions on transport requirements (e.g., ores must travel in rigid-sided vehicles, $\mathrm{CO}_{2}$ must travel in tankers or pipelines).

- Links: Links are entities representing connections between facilities (existing or anticipated connections). Links may be roads, railways, pipelines, etc. Each link can be in one of a set of states expressing transport capacity and a variety of other characteristics. For the CCS optimisation model, IFAP's pipeline-link states map directly to decision variables $z_{e}^{d t}$ and parameters $D, C_{e}^{d}, c_{e}$ and $U^{d}$.

- Facilities: Facilities are entities where goods are produced, consumed, stored or transhipped. Each facility can be in one of a set of predefined states which express capacities and operating costs. Each facility in the IFAP database is of a certain type: for freight, these facility types are processing plants, mines, warehouses, ports and so on. Commodities enter and exit the system at facilities. For CCS, the set of facilities was augmented with types for $\mathrm{CO}_{2}$ sources and sinks. Facility data maps directly to parameters such as $u_{s}$ and the decision variables $y_{c}^{t}$ and $y_{s}^{t}$ in the CCS model.

- Vehicles: Vehicles are required for the transport of some commodities. The term vehicle is used here in a generic sense and can represent a railway train, a flatbed truck, etc. Each vehicle has attributes specifying its capacity and its compatibility with commodities and links. Unlike in road and rail transport, no vehicles are necessary for the transport of $\mathrm{CO}_{2}$.

- Processes: Processes capture and encode the means by which commodities are produced, consumed or transported in the system. There are two process types: logistics processes and transformation processes. Logistics processes encode the characteristics of commodity transportation and storage. A logistics processes relates to a single commodity and states origin(s) and destination(s) for transport. Logistics process definitions incorporate restrictions on transport link types, commodity storage, loading and unloading. Transformation processes consume zero or more input commodities and produce of zero or more output commodities. The ratio of input to output commodities is the primary concern.

Infrastructure planning and analysis focuses primarily on capacity: whether existing capacity is sufficient, whether the capacity of existing infrastructure should be increased, and whether capacity needs to be added by constructing new infrastructure. Thus, the modelling of capacity is a prominent aspect. Capacity and capacity decisions are modelled using states. IFAP's optimization decides on the required capacity by selecting a state for each facility and each link for every time period in the planning horizon. One or more states can be defined for each facility and link (at least one state has to be defined; the initial state, which is permitted to represent "non-existence"). When an entity has more than one state, statetransitions are defined indicating whether it is possible to go from one state to another and, if so, what the cost is. A user is able to model upgrades and construction by defining multiple states for an entity. These original IFAP features are consistent with our specialised CCS network optimisation model, and for the CCS network design application we were able to utilise IFAP's GIS-based user interface and database with only minimal changes and augmentation.

Our reason for analysing CCS networks is to understand how to best increase the infrastructure capacity over time. The utilisation of this capacity is a result of $\mathrm{CO}_{2}$ moving through the system, which, in turn, results from the $\mathrm{CO}_{2}$ transport demand. The demand for $\mathrm{CO}_{2}$ transport is expressed in terms of the mass and/or volume of transport required per period for one or more logistics processes. The CCS network capture and storage sites are modelled as facilities with two states: ('non-capture', 'capture') or ('nonstorage', 'storage'). Pipelines are modelled with $|D|+1$ states, one for the "non-existing" state and a state for each possible pipeline diameter. For CCS modelling there is a single commodity, compressed $\mathrm{CO}_{2}$, and the CCS system operates with specified yearly system-wide demand for $\mathrm{CO}_{2}$ sequestration.

From a user's perspective, the IFAP system is used to enter data and view the details of solutions (e.g., as in Figure 1). The data entry is by way of custom editors and dialog boxes that are implemented in Windows Forms (i.e. Microsoft .NET), bundled into DLLs and called from within the GIS. In general 
terms, the native features of the GIS handle the spatial data, and the custom user interface components handle the CCS model inputs. IFAP is joined by an XML-based interface to an optimiser that solves the model of Section 2 using a commercial integer programming solver.

\section{COMPUTATIONAL STUDY}

Our case study considers only coal-fired power stations and geological sequestration in eastern Australia. These power stations contribute approximately 35\% of Australia's $\mathrm{CO}_{2}$ emissions (Energy Supply Association of Australia (2010)). The goal of the case study is to estimate of the cost of CCS, in dollars per mega-tonne per year, for a given capture target for a 25 year period, and to establish when and where capture and storage facilities and pipelines should be built.

The following assumptions have been made in the preparation of the case study. The $\mathrm{CO}_{2}$ is separated by solvent absorption with $90 \%$ of the emitted $\mathrm{CO}_{2}$ captured; the absorption systems are retrofitted to the power plants. The captured $\mathrm{CO}_{2}$ is compressed to a super-critical phase (at least 8,000 $\mathrm{kPa}$ or 1,160 psi) at the power stations and transported to a regional collection station, from where it is transported to storage sites. The collection pipeline system within emission hubs is not explicitly modeled. The power plants supply power for all separation and compression energy needs. The price of fuel does not change over the planning period. Furthermore, it is assumed that no demand growth and changes in capacity occur during the planning period and that there is no construction lead time.

A number of reports have been used as source material for the model input data: Allinson et al. (2009), ACIL Tasman Pty Ltd (2010), Hayward et al. (2011), Energy Supply Association of Australia (2009), and WorleyParsons (2010). Yearly carbon capture targets $T(f)$ are set using a logistic function that approaches a final yearly carbon target $F$ (in mega-tonnes):

$$
T_{t}=\frac{F}{1+90 e^{-0.35 t}}
$$

where we have used $F=120$ mega-tonnes as the target for the final period in the scenario reported on below. The parameters 90 and 0.35 were selected in this scenario to ensure a smooth transition from 0 to $F$ over the planning horizon.

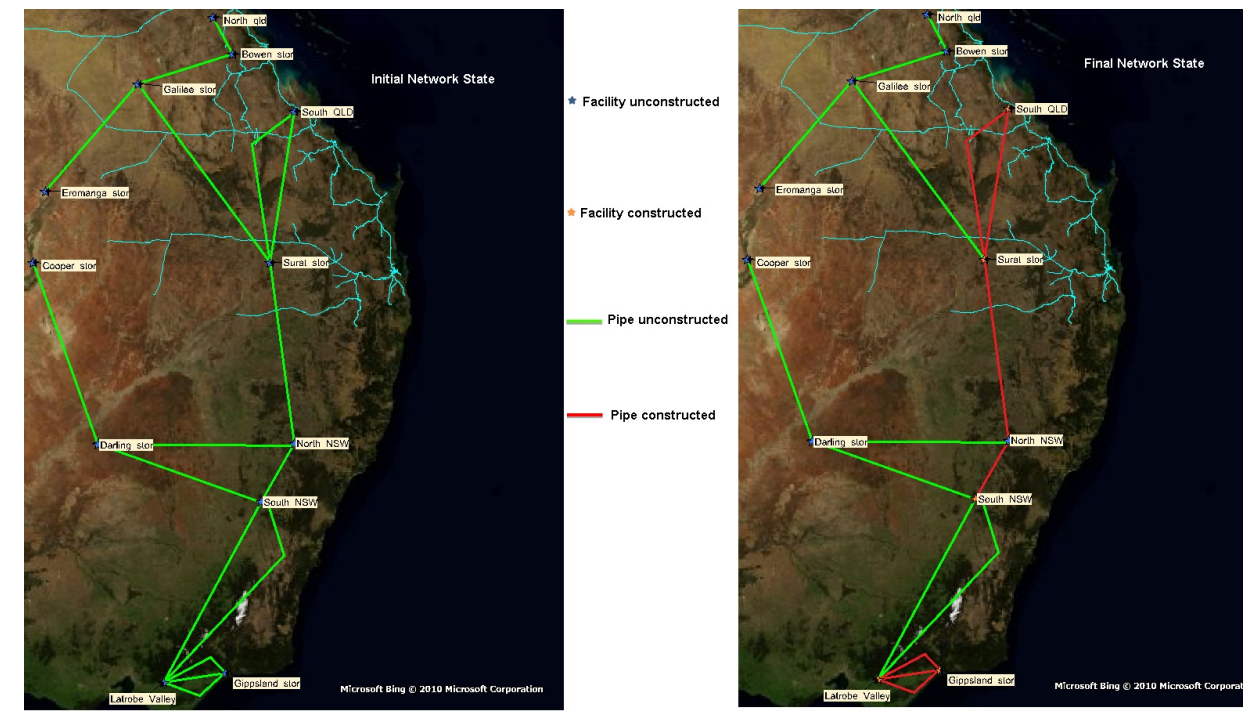

Figure 1. Capture and Storage Sites and Potential Connections

The optimisation model suggests an infrastructure plan in which the first capture and storage facilities as well as the first pipeline are constructed in the first period, connecting the Latrobe Valley power plant to 
the Gippsland basin. The capacity of the connection is increased in the $11^{\text {th }}$ and the $15^{\text {th }}$ time period with the construction of additional pipelines. In the $15^{\text {th }}$ time period, additional capture and storage facilities are also constructed at the power station in Southern Queensland and the Surat basin, respectively, and a pipeline is built connecting these sites. The capacity of this connection is increased in the $20^{\text {th }}$ time period with the construction of additional pipeline. Finally, in the $21^{\text {st }}$ period a capture facility is built at the power station in Southern NSW and it is connected to the Surat basin with pipelines from the Southern NSW location to Northern NSW and then from Northern NSW to the Surat basin. Figure 1 shows the CCS infrastructure at that point in time.

The model allows the increase of transport capacity by the construction of additional pipelines, which exploits the usage of the existing capture and storage sites. Initially, when the carbon capture targets are small, costs are kept low by building just enough transport capacity to accommodate moderate growth. The capital with the most expensive fixed operating costs are constructed as late as possible in the planning horizon. The optimisation model could be modified to disallow the building of multiple pipelines between two sites, if desired. The optimisation model also allows two sites to be connected via an intermediate facility. This flexibility is exploited when the power plant in Southern NSW is connected to the Surat basin. Such indirect connections could also be disallowed if required.

\section{CONCLUSIONS}

Our focus has been on cost minimisation and this ignores many other factors that will contribute to the ultimate success and adoption (or otherwise) of CCS, including public acceptance. Demonstrating that these systems can operate safely in the real world will be important in building public confidence in CCS. Leading up to the time when this is demonstrated, public education may be commenced relating to climate change, energy policies, CCS technologies and their associated risks. We believe that our results provide insights into CCS network designs which, from a deterministic economic perspective, appear most favourable. This kind of information may help guide where public awareness activities and public debates need to be held. This use of the model is in addition to its primary purpose of generating improved CCS cost estimates for input into regional and national scale models of the stationary energy sector.

Deeper and more detailed analysis of infrastructure plans for carbon capture and storage is necessary as part of wider effort to fully assess the potential of CCS to help in cost-effectively reducing greenhouse gas emissions associated with electricity generation. The IFAP system coupled with the CCS optimation module facilitates this type of analysis by providing users with a graphical interface that makes it easy to define scenarios of interest and explore details of the construction schedule. Expanding the functionality of IFAP to accommodate infrastructure planning for pipeline networks proved to be straightforward. More extensive studies are underway with additional scenarios and enhanced optimisation models.

\section{REFERENCES}

ACIL Tasman Pty Ltd (2010). Preparation of energy market modelling data for the Energy White Paper.

Allinson, G., Y. Cinar, W. Hou, and P. R. Neal (2009). The costs of $\mathrm{CO}_{2}$ transport and injection in Australia.

Coal Utilization Research Council (2005). Technology roadmap.

Energy Supply Association of Australia (2009). Electricity Gas Australia 2009.

Energy Supply Association of Australia (2010). Electricity Gas Australia 2010.

Hayward, J. A., P. W. Graham, and P. K. Campbell (2011). Projections of the future costs of electricity generation technologies.

International Energy Agency (2010). World Energy Outlook.

Metz, B., O. Davidson, H. C. de Coninck, M. Loos, and L. Meyer (2005). IPCC special report on carbon dioxide capture and storage. Prepared by Working Group III of the Intergovernmental Panel on Climate Change.

WorleyParsons (2010). DRET CCS Task Force Support - Compression Configuration Study. 